\title{
ON SKEW FIBONACCI MATRICES
}

\section{WEICAI WU1 ${ }^{1}$, JUNJI LIU ${ }^{1}$ and WEIJUN XIE ${ }^{2}$}

${ }^{1}$ School of Mathematics

Hunan Institute of Science and Technology

Yueyang 414006

P. R. China

e-mail: weicaiwu@hnu.edu.cn

${ }^{2}$ School of Primary Education

Hunan Vocational College for Nationalities

Yueyang 414006

P. R. China

\begin{abstract}
In this paper we mainly define the skew Fibonacci sequence and skew Fibonacci matrices, and give the eigenvalues of skew Fibonacci matrices. Furthermore, we present some conditions for a matrix to be a skew Fibonacci matrix.
\end{abstract}

2020 Mathematics Subject Classification: 15A24, 11B39.

Keywords and phrases: skew Fibonacci matrix, eigenvalue, Fibonacci sequence.

Received November 29, 2020

(C) 2020 Scientific Advances Publishers

This work is licensed under the Creative Commons Attribution International License (CC BY 3.0).

http://creativecommons.org/licenses/by/3.0/deed.en_US

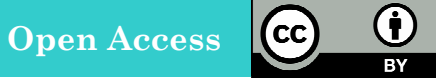




\section{Introduction}

The current paper will focus on skew Fibonacci matrices, which is a cross research target in Fibonacci-Lucas matrices (see [1]), skew Fibonacci sequences and matrices.

Let $\mathbb{Z}=:\{x \mid x$ is an integer $\}, \mathbb{N}=:\{x \mid x$ is an integer, $x>0\}, \mathbb{N}_{0}=: \mathbb{N} \cup\{0\}$, and $\mathbb{C}=:\{x \mid x$ is a complex $\}$. Throughout this paper all matrices we consider below are the $n$ order square matrices over $\mathbb{C}$ without special announcement, $n \in \mathbb{N}$.

Let $f_{1}, f_{2}, \cdots$, denote Fibonacci sequence, i.e., $f_{1}=1, f_{2}=1, f_{n}=f_{n-1}$ $+f_{n-2}, n \geq 3$. Set $f_{0}=0, f_{-i}=(-1)^{i-1} f_{i}, \forall i \geq 1$, then $f_{n}=f_{n-1}+f_{n-2}$ for all $n \in \mathbb{Z}$, and $f_{n}=0$ if and only if $n=0, f_{i}=(-1)^{i-1} f_{-i}$ for all $i \in \mathbb{Z}$.

Let $s_{1}, s_{2}, \cdots$, denote skew Fibonacci sequence, i.e., $s_{1}=1, s_{2}=-1$, $s_{n}+s_{n-1}=s_{n-2}, n \geq 3$. Set $s_{0}=0, s_{-i}=(-1)^{i-1} s_{i}, \forall i \geq 1$, then $s_{n}+$ $s_{n-1}=s_{n-2}$ for all $n \in \mathbb{Z}$, and $s_{n}=0$ if and only if $n=0, s_{i}=(-1)^{i-1}$ $s_{-i}$ for all $i \in \mathbb{Z}$.

It is clear $s_{i}=f_{-i}$ for all $i \in \mathbb{Z}$.

Definition 1.1. Matrix $F$ is called a skew Fibonacci matrix if $F^{2}=-F+E$, where $E$ is a unit matrix.

By Definition 1.1 one obtains $F^{n}+F^{n-1}=F^{n-2}$ for all $n \geq 2$ with $n \in \mathbb{N}$. Using $F^{2}=E-F$ one has $E=F(F+E)$, and hence skew Fibonacci matrices are invertible and $F^{-1}=F+E$. Set $F^{0}=E$, $F^{-n}=\left(F^{-1}\right)^{n}$, then $F^{n}+F^{n-1}=F^{n-2}$ for $\forall n \in \mathbb{Z}$. One can also define skew Fibonacci matrices by $F^{n}+F^{n-1}=F^{n-2}$ for all $n \geq 2$ with $n \in \mathbb{N}$. 


\section{The Main Results}

Let $r=\frac{1+\sqrt{5}}{2}, s=\frac{1-\sqrt{5}}{2}$, then $r^{n}=s_{-n} r+s_{-n+1}, s^{n}=s_{-n} s+s_{-n+1}$, it is clear $\lim _{n \rightarrow+\infty} \frac{s_{n-1}}{s_{n}}=s, \lim _{n \rightarrow-\infty} \frac{s_{n-1}}{s_{n}}=r$.

Lemma 2.1. $f_{n}=f_{m} f_{n-m+1}+f_{m-1} f_{n-m}$ for $\forall n, m \in \mathbb{Z}$.

Proof. When $n=0, f_{m} f_{-m+1}+f_{m-1} f_{-m}=f_{m}(-1)^{m-2} f_{m-1}+f_{m-1}$ $(-1)^{m-1} f_{m}=0=f_{0}$. Assumed that $n \geq 1$. We can show this by induction on $n$. When $n=1, f_{m} f_{2-m}+f_{m-1} f_{1-m}=(-1)^{m-3}\left\{f_{m} f_{m-2}-\right.$ $\left.f_{m-1} f_{m-1}\right\}=(-1)^{m-3}(-1)^{m-1}=1=f_{1}$ by [1, Lemma 1.1(i)]. If $n \geq 2$, $f_{n+1}=f_{n}+f_{n-1}=f_{m} f_{n-m+1}+f_{m-1} f_{n-m}+f_{m} f_{n-m}+f_{m-1} f_{n-1-m}=f_{m}$ $\left\{f_{n-m+1}+f_{n-m}\right\}+f_{m-1}\left\{f_{n-m}+f_{n-1-m}\right\}=f_{m} f_{n-m+2}+f_{m-1} f_{n-m+1}$. Now assume $n \leq-1$. We know $f_{k}=f_{-m+1} f_{k+m}+f_{-m} f_{k+m-1}$ for $\forall k \in \mathbb{N}$, then $f_{-k}=(-1)^{k-1}\left\{(-1)^{m-2} f_{m-1} f_{k+m}+(-1)^{m-1} f_{m} f_{k+m-1}\right\}=(-1)^{m+k-1} f_{m-1} f_{k+m}$ $+(-1)^{m+k-2} f_{m} f_{k+m-1}=f_{m-1} f_{-k-m}+f_{m} f_{-k-m+1}$, let $n=-k$, completing the proof.

Proposition 2.2. If $F$ is a skew Fibonacci matrix, then $F^{n}=f_{-n} F$ $+f_{-(n-1)} E=s_{n} F+s_{n-1} E$ for $\forall n \in \mathbb{Z}$.

Proof. When $n=0, E=s_{-1} E$. Assumed that $n \geq 1$. One can show this by induction on $n$. It is clear when $n=1$. If $n \geq 2, F^{n+1}=F\left(s_{n-1} E\right.$ $\left.+s_{n} F\right)=s_{n-1} F+s_{n} F^{2}=s_{n-1} F+s_{n}(E-F)=s_{n} E+s_{n+1} F$. Now assume $n \leq-1$. One can show $\left(F^{-1}\right)^{m}=s_{-(m-1)} E+s_{-m} F^{-1}$ for all $m \in \mathbb{N}$ by induction on $m$, let $n=-m$, then $F^{n}=s_{n+1} E+s_{n} F^{-1}=s_{n-1} E+s_{n} F$. This proves the Proposition 2.2. 
Remark 2.3. (i) $F$ is a skew Fibonacci matrix if and only if $-F$ is a Fibonacci-Lucas matrix (see [1, Definition 1.2]).

(ii) Assumed that $n \geq 2$, equation $F^{n}=s_{n} F+s_{n-1} E$ can be deduced that equation $F^{n}+F^{n-1}=F^{n-2}$ holds, so one can also define skew Fibonacci matrices by $F^{n}=s_{n} F+s_{n-1} E$.

Proposition 2.4. Assumed that $F$ is a skew Fibonacci matrix. Then

(i) $F^{i}$ are different for distinct $i \in \mathbb{Z}$.

(ii) $x=-r$ or $-s$ if $F=x E, x \in \mathbb{C} ; F^{i}, i \neq 1$ are not skew Fibonacci matrices for all $i \in \mathbb{Z}$.

(iii) The eigenvalues of $F$ are $-r$ or $-s$.

Proof. It is similar to the proof of [1, Proposition 2.3], [1, Proposition 2.4] and [2, Lemma 2.1], respectively.

Proposition 2.5. $s_{-n}=\sum_{i=0}^{n} C_{n}^{i} s_{i}$, i.e., $f_{n}=\sum_{i=0}^{n} C_{n}^{i} f_{-i}$ for $\forall n \in \mathbb{Z}_{0}$.

Proof. When $n=0, s_{0}=\sum_{i=0}^{0} C_{0}^{i} s_{i}=0$. Assumed that $n \geq 1$, then $s_{-n} F+s_{-n-1} E=F^{-n}=\left(F^{-1}\right)^{n}=(F+E)^{n}=\sum_{i=0}^{n} C_{n}^{i} F^{i} E^{n-i}=\sum_{i=0}^{n} C_{n}^{i}\left(s_{i} F\right.$ $\left.+s_{i-1} E\right)=\sum_{i=0}^{n} C_{n}^{i} s_{i} F+\sum_{i=0}^{n} C_{n}^{i} s_{i-1} E$ by Proposition 2.2. We have $\left(s_{-n}-\right.$ $\left.\sum_{i=0}^{n} C_{n}^{i} s_{i}\right) F=\left(\sum_{i=0}^{n} C_{n}^{i} s_{i-1}-s_{-n-1}\right) E$, then $s_{-n}-\sum_{i=0}^{n} C_{n}^{i} s_{i}=0=\sum_{i=0}^{n} C_{n}^{i} s_{i-1}$ $-s_{-n-1}$ for $\forall n \geq 1$ by $r$ or $s$ is an irrational number and Proposition 2.4 (ii). 
Proposition 2.6. Assumed that $F$ is a Fibonacci-Lucas matrix. Then $\sum_{i=1}^{n} F^{i}=F^{n+2}-F^{2}$ and $\sum_{i=1}^{n} F^{2 i-1}=F^{2 n}-E$ for $\forall n \in \mathbb{N}$.

Proof. One can show $\sum_{i=1}^{n} f_{i}=f_{n+2}-1, \sum_{i=1}^{n} f_{2 i-1}=f_{2 n}$ and $\sum_{i=1}^{n} f_{2 i}=$ $f_{2 n+1}-1$ by induction on $n$, the other is clear by the simple verification since Proposition 2.2.

Lemma 2.7. (i) If $F_{1}+F_{2}=-E$, then $F_{1}$ is a skew Fibonacci matrix if and only if $F_{2}$ is a skew Fibonacci matrix.

(ii) If $F$ is a skew Fibonacci matrix, then the NI matrix ([2]) and the transpose matrix of $F$ are skew Fibonacci matrices.

Proof. (i) It is clear $F_{1}=-E-F_{2}$, then $\left(-E-F_{2}\right)^{2}+\left(-E-F_{2}\right)-$ $E=F_{2}^{2}+F_{2}-E$.

(ii) Set $F_{1}+F=-E$, by (i) one obtains $F_{1}$ is a Fibonacci-Lucas matrix and $F_{1}=-E-F=-F^{-1}$. Consider $F^{\prime}, 0=\left(F^{2}+F-E\right)^{\prime}=\left(F^{\prime}\right)^{2}$ $+F^{\prime}-E$.

Let $f(F)$ be any polynomial of matrix $F$ on $\mathbb{C}$.

Proposition 2.8. (i) Assumed that $F$ is a Fibonacci-Lucas matrix. Then $f(F)$ is a Fibonacci-Lucas matrix if and only if $f(F)$ appears in one of the following: (1) $f(F)=r E$ or $s E$; (2) $f(F)=F$; and (3) $f(F)=-F+E$.

(ii) Assumed that $F$ is a skew Fibonacci matrix. Then $f(F)$ is a skew Fibonacci matrix if and only if $f(F)$ appears in one of the following: (1) $f(F)=-r E$ or $-s E$; (2) $f(F)=F$; and (3) $f(F)=-F-E$. 
Proof. (ii) is similar to (i). (i) We only need to consider $a F+b E$ for $\forall a, b \in \mathbb{C}$ by [1, Proposition 2.1]. Let $(a F+b E)^{2}-(a F+b E)-E=\left(a^{2}+2\right.$ $a b-a) F+\left(a^{2}+b^{2}-b-1\right) E=0$. If $a^{2}+b^{2}-b-1=0$, then $a^{2}+2 a b$

$-a=0$, we have $a=0, b=r$ or $s$; or $a=1, b=0$; or $a=-1, b=1$. If $a^{2}+b^{2}-b-1 \neq 0$, then $a^{2}+2 a b-a \neq 0$, we have $F=r E$ or $s E$ by [1, Proposition 2.4], the other is clear by the simple verification.

\section{Acknowledgement}

Weicai Wu acknowledges project supported by the Research and Innovation Team of Hunan Institute of Science and Technology (Grant No. 2019-TD-15) and A project supported by Scientific Research Fund of Hunan Provincial Education Department (Grant No. 19C0860).

\section{References}

[1] Weicai Wu, 2 order Fibonacci-Lucas matrices, Far East Journal of Mathematical Sciences 124(1) (2020), 1-5.

DOI: http://dx.doi.org/10.17654/MS124010001

[2] Weicai Wu, 3 order Fibonacci-Lucas matrices, Universal Journal of Mathematics and Mathematical Sciences 13(1) (2020), 33-39.

DOI: http://dx.doi.org/10.17654/UM013010033 